\title{
Der Soziologe, der Arzt und die Diagnose alläglichen Leidens in der neoliberalen Marktgesellschaft ${ }^{1}$
}

«Nicht verlachen, nicht bemitleiden, nicht verabscheuen, sondern verstehen ...»

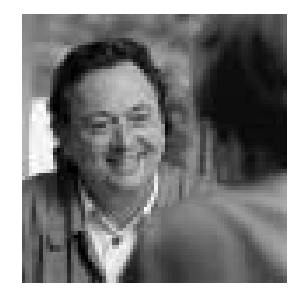

In der neoliberalen Marktgesellschaft weht ein kalter Wind. Er trifft besonders jene ungeschützt, die aufgrund körperlicher oder seelischer Schwächen verwundbar, durch ihre Familiensituation weniger flexibel und mobil als andere, aufgrund ihres Alters nicht mehr employable erscheinen. Arzt und Soziologe beobachten ein zunehmendes Leiden an dieser entfesselten Konkurrenzlogik und sollten hiervon öffentlich Zeugnis ablegen.

Un vent froid souffle sur la société de consommation néolibérale. Il éprouve particulièrement ceux qui sont déjà fragiles physiquement ou moralement, ceux que leur situation familiale rend moins souples ou moins mobiles que d'autres, ou ceux qui ne sont plus susceptibles de trouver un emploi du fait de leur âge. Les médecins et les sociologues observent les maux grandissants liés à cette logique imparable de la concurrence et devraient en donner un témoignage public.

Franz Schultheis

\section{Der Arzt als Soziologe - der Soziologe als Arzt}

Arzt und Soziologie haben nicht oft Gelegenheit zum Meinungsaustausch, bzw. sie nutzen diese nicht regelmässig. Dabei haben sie sehr vieles gemein und könnten aus einem solchen Austausch einigen Gewinn ziehen. Der Soziologe trifft nämlich, wenn er sich der alltäglichen Lebenswelt und den gewöhnlichen Problemen seiner Mitmenschen zuwendet, auf eben denselben Herrn Meyer und die gleiche Madame Dupont, die man auch im Wartezimmer der Arztpraxis antrifft. Dorthin kommen sie, um über ihre gesundheitlichen Sorgen und körperlichen Gebrechen zu sprechen, und hoffen auf Rat und Abhilfe für ihre Leiden und Nöte. Zum Soziologen kommen sie nicht in die Sprechstunde, sondern dieser begibt sich dahin, wo Herr Meyer und Frau Dupont wohnen, arbeiten oder ihre Freizeit verbringen, um mit ihnen über ihre Lebensverhältnisse, Freuden, Erschwernisse und oft vielfältigen Leiden zu reden, Beobachtungen anzustellen und zu Diagnosen über die Ursachen und Zusammenhänge dieser Befindlichkeiten zu gelangen.

\section{Soziokulturelle Einflüsse auf die individuelle Gesundheit}

Das dem Soziologen mitgeteilte Leiden scheint zunächst weit von Gallensteinen, Bluthochdruck, Hautentzündungen und Verdauungsproblemen entfernt zu sein, mit denen es der Arzt tagtäglich zu tun hat; handelt es sich doch bei jenem um wirtschaftliche, gesellschaftliche oder kulturelle Fragen und um Probleme wie Arbeitslosigkeit, Vereinsamung, Stress und Druck am Arbeitsplatz, um Familien- und Ehekrisen, schwierige Wohnverhältnisse, Entwurzelungen in Folge von Migration, um Erfahrungen von Macht und Ohnmacht in der Arbeitswelt oder im Umgang mit Bürokratien, um Rassismus und Vorurteile, Delinquenz, schulisches Versagen oder was auch immer.

Nur wissen Ärzte ebenso gut wie Soziologen, dass alle die genannten gesellschaftlichen Faktoren und Fragen durchaus einen indirekten oder gar direkten Einfluss auf die Gesundheit der Menschen haben können. Sie beide wissen ebenso gut, dass Scheidungen und Familienkrisen nicht nur mit erhöhter

\footnotetext{
1 Der vorliegende Beitrag basiert auf einem Vortrag, der unter dem Titel «Gabe und Gegengabe - die elementare Form sozialer Beziehungen im Zeitalter des homo oeconomicus» anlässlich des 26. Schweizer Kongresses für Allgemeine Medizin in La Chaux-de-Fonds im September 2003 gehalten wurde.
} 
Selbstmordgefahr, sondern auch mit vielfältigen, die Gesundheit gefährdenden Faktoren einhergehen können. Die Ärzte wissen noch viel besser als der Soziologe, welche Folgen - in Form psychosomatischer Leiden - dauerhafter Arbeitsstress, ständige Lärmbelästigung und Überforderung im Beruf hervorbringen können. Sie wissen auch, dass soziale Ungleichheiten, Armut, soziale Ausgrenzung etc. mit einer deutlich höheren Krankheitsanfälligkeit, erhöhtem Alkohol- und Drogenmissbrauch einhergehen und dass die durchschnittliche Lebenserwartung von Menschen in den wenig qualifizierten und prekären Berufsgruppen, statistisch besehen, bis zu sieben Jahre kürzer ist als etwa bei einem Grundschullehrer.

Und da die rund 10\% der Schweizer Bevölkerung, die nach den Kriterien der OECD offiziell als arm gelten, auch etwa 10\% ihrer Patienten darstellen bzw. aufgrund ihrer grösseren Krankheitsanfälligkeit überdurchschnittlich viel Zeit und Raum in der Alltags-«Praxis» einnehmen, wissen Ärzte eben auch aus nächster Nähe, wie Armut und Elend aussehen und sich anfühlen. Arzt und Soziologe teilen sich demnach weitgehend ein- und denselben «Gegenstand»: den Menschen. Sie sind Menschenwissenschaftler, und ihre beruflichen Aufgaben und Tätigkeiten gehen zu einem guten Teil von gemeinsamen Fragestellungen aus.

\section{Gemeinsame Grundhaltung und gleiche Erwartungen}

Mehr noch: auch die beim Umgang mit diesen Mitmenschen vorausgesetzte Grundhaltung sollte eigentlich von ihren ethischen Prämissen und Implikationen her beim Arzt und Soziologen ganz ähnlich beschaffen sein; und man kann in einer moralischen Grundregel der Philosophie Spinozas einen gemeinsamen Nenner dafür finden, wie man als Arzt oder als Soziologe dem anderen, der uns von seinen Leiden und Sorgen Zeugnis ablegt, zu begegnen hat. ${ }^{2}$ Spinozas ethische Maxime lautet:

Nicht verlachen, nicht bemitleiden, nicht verabscheuen, sondern verstehen!

Zum Arzt oder zum Soziologen geht man nicht, um diese mit Erzählungen über Nöte, Missgeschicke und Probleme zu unterhalten oder gar zu amüsieren

2 Vgl. hierzu im einzelnen: Bourdieu, P. u.a.: Das Elend der Welt, Konstanz, Universitätsverlag 1997 und die Ausführungen im Nachwort von Franz Schultheis. und danach mit süffisanten oder moralinsauren Randbemerkungen versehen auf den Heimweg geschickt zu werden. Man wendet sich an sie, um Gehör und Aufmerksamkeit zu finden, das Gefühl zu bekommen, dass man ernst genommen wird und dass die vorgetragenen Sorgen, die einem das Leben schwer machen und die man oft mit kaum jemand anderem teilen kann, hier zumindest mitgeteilt werden können, ohne dass man zum Schaden auch noch den Hohn bekommt.

\section{Menschen im Mahlstrom entfesselter Marktkonkurrenz}

\section{Jenseits "einverleibter» gesellschaftlicher Strukturen und Rahmenbedingungen ...}

Eine zentrale Quelle des Leidens an gesellschaftlichen Lebensbedingungen findet sich im raschen, radikalen oder gar revolutionären Wandel von gesellschaftlichen Strukturen und alltäglichen Lebensverhältnissen. Dieser schlägt sich in einem Bruch zwischen den vom Menschen internalisierten bzw. sprichwörtlich «einverleibten» gesellschaftlichen Strukturen (dem Ensemble an Denkschemata, moralischen Standards und Verhaltensgewohnheiten, die der Soziologe «Habitus» nennt) und den Rahmenbedingungen der gesellschaftlichen Umwelt nieder und produziert das, was der Soziologe Anomie nennt, d.h. einen Mangel an normativen Koordinaten und Orientierungen, an denen man seine Erwartungen und Handlungen mit einiger Verlässlichkeit ausrichten kann. Genau hiervon wird im folgenden die Rede sein. Wir leben heute in einer Zeit massiver wirtschaftlicher, sozialer und kultureller Umbrüche. Darüber besteht bei allen Experten der Sozial-, Wirtschafts- und Humanwissenschaften ein ausgeprägter Konsens, und man ist sich auch einig darin, dass dieser radikale Wandel ungefähr in den Jahren 1980 begonnen hat. Nur wählt man für die Kennzeichnung dieses Wandels sehr unterschiedliche Konzepte und spricht von Globalisierung, post-industrieller Gesellschaft oder Neoliberalismus.

\section{... verändert die neue Marktordnung die normativen Anforderungen ...}

Im Rahmen dieses Wandels kommt es, nach dem in zahlreichen sozialwissenschaftlichen Publikationen dokumentierten Stand der Forschung, zu einer grundlegenden Veränderung der normativen Anforderungen an den Arbeitnehmer bzw., um es zeitgemäss auszudrücken, an das heutige Humankapital. Der im Zeitalter des Neoliberalismus geforderte und geförderte ökonomische Habitus des marktfähigen Arbeitnehmers lässt sich dank der Analyse der ein- 
schlägigen Managementliteratur durch Boltanski und Chiapello [1], aber auch aufgrund eigener Forschungen des Autors folgendermassen idealtypisch skizzieren und profilieren.

Seit den 80er Jahren setzen sich in der Managementliteratur auf breiter Front ein neuer Diskurs und ein neues Vokabular durch, mittels deren die vom Arbeitnehmer erwarteten Qualitäten beschrieben werden.

$\mathrm{Zu}$ diesen immer mehr eingeforderten bzw. vorausgesetzten Merkmalen und Kompetenzen zählen (präsentiert in alphabetischer Reihenfolge) vor allem:

Autonomie

Charisma

Employability

Flexible Einsatzfähigkeit

Geselligkeit

Impulse geben können

Innovatorisch sein

Kommunikationsfähigkeit

Kompromissfähigkeit

Kreativität

Lebenslanges Lernen

Mitreissen können

Mobilität

Neugierde

Offenheit

Plurikompetenz

Projektmanagement

«Radar» für Informationen

Risikobereitschaft

Selbstevaluation

Selbstmanagement

Selbstsicherheit

Soziales Kapital schöpfen

Spontaneität

Toleranz

Verfügbarkeit

Vermittlerrolle

Vernetzung

Vielfalt der Projekt

Visionär sein

Zuhören können

... an den marktfähigen Arbeitnehmer ...

Gemeinsamer Nenner dieses Steckbriefs des idealen Arbeitnehmers scheint zu sein:

- er arbeitet ständig und lebenslang an der Perfektionierung oder zumindest Bewahrung seines «Humankapitals» in Gestalt seines inkorporierten kulturellen und sozialen Kapitals,

- er denkt und handelt im Rahmen von je befristeten und begrenzten Projekten statt in Dimensionen lebenslanger beruflicher Karrierevorstellungen,
- er situiert sich im Kontext personengebundener sozialer Netzwerke (seinem «sozialen Kapital» an aktivierbaren Ressourcen an Unterstützung), statt auf institutionalisierte Netzwerke zu bauen.

Der «employable man»

- orientiert sich an seinem eigenen, in Gestalt von konkreter Nachfrage messbaren Marktwert, statt nach einem dauerhaften Status zu streben, und

- begnügt sich mit einer konjunktur- und situationsabhängigen Lebensführung, anstatt sich an einen langfristigen Lebensentwurf zu klammern.

- Er ist insofern in seiner Lebensorientierung stärker aussengeleitet als das klassische bürgerliche Individuum mit starker Innenleitung.

- Der marktgängige Arbeitnehmer ist geographisch mobil und beruflich flexibel und weiss dies mit seinen privaten Lebensarrangements in Einklang zu bringen, welche dadurch tendenziell auch den Charakter von zeitlich begrenzten Projekten annehmen. ${ }^{3}$

\section{... entsprechend den allgemeinen Grundzügen der neoliberalen Wirtschafts- und \\ Gesellschaftslehre, ...}

Der beschriebene ökonomische Habitus des «employable $\operatorname{man}^{4} »$ widerspiegelt direkt die allgemeinen Grundzüge der neoliberalen Wirtschafts- und Gesellschaftslehre.

Hierzu zählen Aspekte wie:

- Optimierung der Markt-Transaktionen (Zahl und Frequenz)

- Optimierung der Vertragsabschlüsse (Zahl und Frequenz)

- Reduktion des Inhalts von Verträgen auf die effektiv $\mathrm{zu}$ erbringende Leistungen (reiner Leistungslohn und reiner Zweckkontrakt)

- Glaube an die rationale und moralische Notwendigkeit des Marktes

- Ausdehnung und Intensivierung des Markthandelns im gesellschaftlichen Raum

- Ausräumen aller dabei im Wege stehenden Schranken

- Schaffung von Quasi-Märkten bzw. Sub-Märkten auch im Innern ein- und desselben Unternehmens

3 Man wird schon gemerkt haben, dass auch das Leben des erfolgreichen Marktmenschen alles andere als reines Honigschlecken ist. Man fordert ihm ein sehr hohes (allzu hohes?) Mass an Selbstkontrolle und Selbstdisziplin ab, eine Dauerreflexivität, die irgendwann Ermüdungserscheinungen und eine «Fatigue d'être soi», wie es der treffende Titel eines Buches von Ehrenberg auf den Nenner bringt, zeitigen muss.

4 Hier wie andernorts wird «man» geschlechtsneutral in seiner anthropologischen Bedeutung verwendet. 
- Glaube an die Überlegenheit der Marktgesellschaft als Ergebnis eines offenen Prozesses

- Glaube an den Unternehmer als Prototypen eines sensibel und rational auf die Marktkräfte reagierenden Akteurs und

- Forderung nach einer Orientierung an diesem rational wie moralisch überlegenen Modell für jedermann

So weit der in vielfältigen sozialwissenschaftlichen Studien diagnostizierte Wandel der modernen Arbeitsgesellschaft auf makrostruktureller (Wirtschaftsstrukturen) wie individueller Ebene (Habitus).

\section{... wie ein Beispiel moderner Umstrukturierung zeigt:}

Unser Beitrag wird nun den Versuch machen, diesen vor unseren Augen stattfindenden gesellschaftlichen Wandel und seine Folgen für die betroffenen Menschen anhand der Beobachtungen und Analysen der Veränderungen in einem traditionsreichen Schweizer Unternehmen von Weltruf näher zu beleuchten. Es handelt sich um ein Traditionsunternehmen, das in den 90er Jahren seine Unternehmenskultur im Gefolge einer zweifachen Fusion radikal umkrempelte bzw. «modernisierte» und im gleichen Schritt rund 4000 Mitarbeiter entliess bzw. wie man dort sagt: «freigestellt hat». Hierbei sei gleich einleitend angemerkt, dass dieses Unternehmen bis zu diesem Zeitpunkt einen besonderen Stolz darin zu finden schien, noch nie einen Mitarbeiter vor die Tür gesetzt $\mathrm{zu}$ haben. Es handelt sich also um eine tief greifende Metamorphose, nach welcher dieses Unternehmen auch und gerade für langjährige Mitarbeiter aller hierarchischen Positionen schlicht nicht mehr wiederzuerkennen war.

Der Autor hatte Gelegenheit, mit einer Gruppe jüngerer Soziologen und Soziologinnen diesen Prozess über eine Dauer von mehr als zwei Jahren beobachtend $\mathrm{zu}$ begleiten und $\mathrm{zu}$ erforschen. ${ }^{5}$ Die Befunde und Ergebnisse dieser konkreten Beobachtungen und Analysen können unseres Ermessens über den Einzelfall hinaus sehr treffend als Spiegel unserer Gesellschaft auf dem Wege in ein neues, neoliberales Zeitalter dienen.

Im Zentrum hierbei steht die Frage nach dem Menschen: Was wird aus ihm, dem Homo sapiens, unter den Bedingungen einer brutalen Radikalisierung der Marktlogik? Entwickelt er sich vollends zu einem Homo oeconomicus, wie es die moderne Wirtschaftslogik zu fordern scheint und viele der beobachtbaren Veränderungen es bereits andeuten? Gibt es Widerstände und Kräfte der Beharrung? Wie wird dieser Wandel konkret erlebt und erlitten? Mit solchen gewollt «naiv» erscheinenden Fragen folgen wir einem zentralen soziologischen Erkenntnisinteresse, welches von Max Weber in der folgenden Weise auf einen klaren Nenner gebracht wurde: «Ausnahmslos jede, wie immer geartete Ordnung der gesellschaftlichen Beziehungen ist, wenn man sie bewerten will, letztlich auch darauf hin zu prüfen, welchem menschlichen Typus sie, im Wege äusserer oder innerer (Motiv-)Auslese, die optimalen Chancen gibt, zum herrschenden zu werden.» [2]

\section{Ein nicht wiederzuerkennendes Unternehmen}

1997 bekamen wir die Möglichkeit, den grundlegenden Wandel eines Schweizer Traditionsbetriebs aus nächster Nähe zu beobachten und zu analysieren. Nach einer Fusion mit einem anderen inländischen und nachfolgend mit einem ausländischen Unternehmen der gleichen Branche wurde diese $\mathrm{Zu}$ sammenlegung für die Durchsetzung einer weitreichenden und tiefgehenden Rationalisierung und Modernisierung genutzt. $\mathrm{Zu}$ diesem Zwecke holte man sich eine weltweit operierende Unternehmensberatung ins Haus, die den Plan für eine Totalrenovation ausarbeitete und umsetzte.

\section{Employability oder der Wettkampf um das Verbleiben im neuen Unternehmen}

Möglichst viele Arbeitsplätze sollten eingespart werden und hierbei legte man die Elle der Employability an, um die rund 4000 Überzähligen, sozusagen den Ausschuss des Modernisierungsprozesses, auszusortieren. Das Prinzip der Employability wurde dabei nach den Plänen der für den Umbau zuständigen Consultants in Form von Workshops umgesetzt: alle Mitarbeiter der fusionierenden Unternehmen wurden eingeladen, an ausgewählten Tagungsorten einen Wettkampf um ihr Verbleiben bzw. Überleben $\mathrm{zu}$ bestehen und dabei zu beweisen, dass sie gegenüber ihren Konkurrenten ein höheres Mass an Employability aufzuweisen hatten. ${ }^{6}$

\footnotetext{
5 Eine ausführliche Präsentation der Fragestellungen, Methoden und Befunde dieser Forschung wird die in Kürze abgeschlossene Doktorarbeit von Andrea Buss-Notter bieten.

6 Es bedarf wohl kaum einer längeren Fussnote, um auf die besondere Brutalität dieser Form sozialer Auslese zu verweisen. Das sozialdarwinistische Prinzip des «survival of the fittest» wird unter den Bedingungen solcher «Überlebensübungen» ohne Umschweife zelebriert, und analog zu den traditionellen Ausscheidungskämpfen (von den Gladiatorenkämpfen im Zirkus des alten Rom bis hin zum modernen Sport oder medial inszenierten Wettbewerben aller Art) lässt sich auch hier die kollektive Illusion wahren, dass alles mit rechten Dingen zugeht.
} 


\section{Der grosszügige Sozialplan unter der Lupe ...}

Um die Verlierer nicht einfach auf der Strasse sitzen $\mathrm{zu}$ lassen (was für das Image des Unternehmens wenig erfreuliche Schatten werfen könnte), entwarf das Unternehmen einen recht grosszügigen Sozialplan, und dieser gab denn auch den Rahmen dafür ab, Soziologen auf den Plan zu rufen. Die mit dem «sozial verträglichen» Personalabbau beauftragte Gruppe von Kadern des Unternehmens liess uns Einblick in ihre Arbeit nehmen und ermöglichte es uns, die sozialen und menschlichen Folgekosten der vonstatten gehenden Rationalisierung zu beobachten und zu analysieren.

Unsere Forschung bediente sich unterschiedlicher Methoden wie:

- Teilnehmende Beobachtung

- Auswertung schriftlicher Quellen unterschiedlichster Herkunft und Funktion, Personalakten, Trainingsprogramme, Pläne des Consulting-Unternehmens etc.

- Statistische Auswertung von Personalakten (soziodemographische Profile der Population freigestellter Mitarbeiter nach soziologisch relevanten Kriterien wie Alter, Herkunft, Familienstand etc.)

- Qualitative Interviews mit mehr als 100 Betroffenen unterschiedlichster Statuspositionen im Unternehmen

\section{Die Folgen der Restrukturierung aus der "Froschperspektive» der Betroffenen}

Vor allem bei den qualitativen Interviews ging es darum, die menschlichen Erfahrungen mit dem erlebten Umbruch aus der Sicht der Betroffenen ans Licht zu bringen und deren Zeugnisse einzuholen und festzuhalten. Dieses Kernstück des Projektes diente u.a. auch dem Zweck, den Wandel des Unternehmens, welcher in künftigen Zeiten wohl hauptsächlich aus der Vogelperspektive der Unternehmensspitze als einschneidender Erfolg einer Anpassung an die Zwänge des Marktes und verschärfter globaler Konkurrenz gefeiert werden wird, auch aus der Froschperspektive des ganz normalen Angestellten zu sehen und deutlich zu machen, dass es sich bei diesem Unternehmenswandel um eine sehr doppelschneidige Angelegenheit handelt. Dieser bringt nicht nur auf seiten der ihn passiv wie einen Schicksalsschlag erleidenden Alltagsmenschen enorme Verlustgefühle und Leiden mit sich, sondern destabilisiert auch das Verhältnis der im Unternehmen Verbleibenden dauerhaft, zerstört langfristig das
Vertrauen, das der einfache Arbeitnehmer in es setzte, und schlägt sich im sog. Survivor-SicknessSyndrom nieder, welches wohl auch für das Unternehmen selbst langfristig negative Konsequenzen in Form von sinkender Identifikation (die viel beschworene Corporate identity) und Arbeitsmotivation haben kann.

\section{Typische "Gewinner» und "Verlierer»?}

Mittels der statistischen Auswertung der demographischen und sozialen Merkmale der rund 4000 aussortierten Menschen, der Überzähligen, konnten wir zunächst der Frage nachgehen, ob denn der postulierte Wettkampf ums Überleben tatsächlich so egalitär wie behauptet vor sich ging oder ob es typische Verlierer und typische Gewinner-Profile gab.

\section{Wer sind die "Verlierer»?}

Schauen wir uns zu diesem Zweck einmal das Profil der «Verlierer» bei dieser Ausscheidungskonkurrenz an. Fragt man nach den persönlichen Merkmalen und Profilen, die den Arbeitnehmer der fusionierenden Unternehmen für eine Freistellung prädisponierten, in gewissem Sinne also nach «Handicaps» in der Konkurrenz um knappe (bzw. aus Rationalisierungsgründen in der Logik der Shareholder value künstlich verknappte) Stellen, so stösst man zunächst auf den Faktor Geschlecht.

Frauen waren bei der Population ausgesonderter Mitarbeiter, den «Leavern», weit überrepräsentiert, was zu einem guten Teil mit einem weiteren Typus des Handicaps bzw. der sozialen Verwundbarkeit zusammenhängen dürfte: der Familiensituation. Wie unsere statistische Analyse offen legte, scheinen Mütter von Kleinkindern, aber auch noch schulpflichtigen Kindern in ihrer vom Unternehmen evaluierten Employability stark beeinträchtigt (gehandicapt) und wurden weit überdurchschnittlich freigestellt ${ }^{7}$, während Vätern in gleichen Familienverhältnissen ein solches Schicksal als «strukturelle Opfer» der Modernisierung erspart blieb. Kinder als Handicap? Glaubt man, dass unsere fortgeschrittenen Industriegesellschaften in Sachen Geschlechterungleichheit doch einige Fortschritte gemacht hätten, so wird

7 Hier sei darauf verwiesen, dass auch Teilzeitarbeit ein deutlicher Indikator für die Verwundbarkeit von Angestellten im sozialen Selektionsprozess ist, allerdings signifikant mit den beiden genannten Faktoren weibliches Geschlecht und Familienlasten korrelierte und in dieser Weise für eine Ausgrenzung der dreifach Betroffenen prädestinierte. 
man angesichts aktueller sozioökonomischer Veränderungen schnell eines Besseren belehrt.

Weiterhin erwies sich der Faktor Alter als zentrales Selektionskriterium: Bereits ab 45 Jahren wurden Arbeitnehmer signifikant häufiger freigestellt, ab 55 Jahren aber prinzipiell von einer Frühverrentung betroffen, eine Entscheidung, die aufgrund ihrer für das Unternehmen katastrophalen Folgen schnell revidiert wurde. ${ }^{8}$ Auch das kulturelle Kapital in Form von schulischen und universitären Diplomen erwies sich als einschneidendes Selektionsprinzip: je geringer das schulische Kapital, desto grösser die Ausscheidungswahrscheinlichkeit - so einfach lesen sich die aus der statistischen Auswertung der Profile von Gewinnern und Verlierern destillierten Kriterien.

Kommt noch hinzu ein geographisches bzw. sozialtopographisches Selektionselement, welches die spezifischen historisch gewachsenen Verhältnisse der Schweiz gut widerspiegelt: Arbeitnehmer aus den französisch- und italienischsprachigen Regionen der Schweiz waren deutlich häufiger dem Risiko des Arbeitsplatzverlustes ausgesetzt als jene aus der Deutschschweiz, und das sich in dieser Form der Sonderung zum Ausdruck bringende Prinzip der Dominanz des Zentrums (die Deutschschweiz als zentraler Wirtschaftsstandort) spielte auch innerhalb dieses dominanten Sprachraums nochmals nach der Logik der Zentralität (Zürich als Kapitale des Kapitals der Schweiz war der Ort, an dem es sich am ehesten «überleben» liess, Basel-Land oder die Ostschweiz hingegen gaben schlechte Standorte im Rennen ums Verbleiben ab).

\section{Gesundheitszustand und «Markt- fähigkeit» des Arbeitnehmers - vom solidarischen Füreinander zur kollektiven Intoleranz}

Kommen wir nun aber zu einem gerade für den Arzt als besonders angesprochenem Leser dieser Zeilen wichtigen Aspekt der Analyse des Leidens an aktu-

8 Hier wie auch an vielen anderen Orten muss der Soziologe schlicht seinem Erstaunen Ausdruck geben und gestehen, immer wieder davon überrascht $\mathrm{zu}$ werden, mit welcher Weltfremdheit und Naivität hoch bezahlte Consultants wider allen gesunden Menschenverstand ihre am grünen Tisch der Wirtschaftshochschulen erworbenen abstrakten Modellierungskünste contrafaktisch der Wirklichkeit verschreiben bzw. oktroyieren wollen. Eigentlich müsste man wissen, dass man das soziale Gedächtnis eines Unternehmens zerstört, wenn man eine ganze Generation schlicht vergessen macht. Aber gesellschaftliche Eliten funktionieren nun einmal nicht nach Prinzipien des gesunden Menschenverstandes. ellen gesellschaftlichen Veränderungsprozessen. Innerhalb der Gruppe der ausgesonderten «unemployable (wo-)men» des Unternehmens trafen wir viele Personen an, die oft seit geraumer Zeit mit gesundheitlichen Problemen, seien sie körperlicher oder seelischer Art, zu kämpfen hatten.

Hier begegneten wir Mitmenschen, die uns von ihrem vermeintlich individuellen Schicksal berichteten und in Wirklichkeit Zeugnis von einer Renaissance der kollektiven Intoleranz gegenüber Schwächen und Makeln aller Art ablegten. Ihre Handicaps, bis dahin im Rahmen einer paternalistischen Unternehmenskultur toleriert und unproblematisch in den Arbeitsalltag integriert, reichten von Krebsleiden bis hin zu besonderer Stressanfälligkeit - persönliche Schicksale, für die im Unternehmen durchaus «Platz» war und die von den Arbeitskollegen solidarisch aufgefangen und seitens verständnisvoller Vorgesetzter «normalisiert» werden konnten. Viele der Betroffenen des Stellenabbaus sprachen vom früheren Unternehmen als einer grossen «Familie», der sich die Mitarbeiter, ob auf bedeutenden Positionen oder in unteren Rängen, zugehörig fühlten; und in einer solchen «Familie» werden schwache Mitglieder nicht einfach ausgesondert. Anders jedoch, wenn die einzig einer ökonomischen Rationalität unterworfene Maxime lautet: Geschäft ist Geschäft und ein Unternehmen ist keine karitative Einrichtung, weshalb Gefühlsduseleien fehl am Platz sind.

\section{Marktfähigkeit des Unternehmens versus eine aufs Spiel gesetzte interne Loyalität}

Hier wird gar nicht in Frage gestellt, dass ein solches Unternehmen, wie jedes andere, Zwängen der Konkurrenzfähigkeit unterworfen ist und am Kriterium der Rentabilität nicht vorbeisehen kann. Gleichzeitig aber ist $\mathrm{zu}$ bedenken, dass Unternehmen auf die Loyalität (das gute alte Wort «Treue» klingt wohl etwas antiquiert, ist aber treffend), Zuverlässigkeit und Identifikation ihrer Mitarbeiter zählen müssen und neben dem eigentlichen Arbeitsvertrag sozusagen noch ein stillschweigender «Statusvertrag» abgeschlossen wird, nach welchem man sich Unterstützung in guten wie in bösen Zeiten schuldet. Selbstverständlich kommen Unternehmen in Krisenzeiten oft nicht daran vorbei, Personal einzusparen und schmerzliche Einschnitte vorzunehmen. Wie aber sollte man es gegenüber langjährigen Mitarbeitern und sich selbst gegenüber rechtfertigen können, wenn man in Zeiten hoher Unternehmenserträge und bester Bilanzen Arbeitsplätze wegrationalisiert und dem goldenen Kalb der Shareholder value als Opfer darbringt? 


\section{Sozialer Identitätsverlust als Quelle neuer Krankheiten}

Geopfert werden konkrete Mitmenschen, die an das Unternehmen glaubten und emotional an es gebunden waren, in ihm ihre Heimat erblickten und ihre soziale Identität sehr weitgehend auf der Identifikation mit ihm bauten. Auch wenn häufig bei den Opfern dieser Rationalisierung keine sehr tiefgehende materielle Not die Folge war, viele eine neue Stelle fanden oder qua Frührente oder Outplacement weiterhin materiell gesichert blieben, so beschränkt sich Not, Leiden und Elend nun einmal nicht alleine auf die ökonomische Seite des Lebens. Für den Soziologen, und wohl auch den Arzt, zählen ebenso die immateriellen, symbolischen oder moralischen Nöte des Menschen und ihr Niederschlag in physischen oder psychischen Leiden. Der Verlust von Heimat, sozialer Bindung und Einbindung, Solidarität mit den Arbeitskollegen und Stolz auf die Unternehmenszugehörigkeit ist eine sehr ernst zu nehmende Quelle der Verwundbarkeit und Destabilisierung, von existentiellen Nöten. Ärzte und Soziologen wissen aus ihrer tagtäglichen Erfahrung heraus, mit welchen Begleit- und Folgeerscheinungen diese Verwundbarkeit einhergehen kann und welche enormen individuellen und gesellschaftlichen Kosten von solchen unternehmerischen Entscheidungen verursacht werden.

Während sich Wirtschaftsunternehmen vermehrt in ökologischen Fragen sensibilisiert und aufgeschlossen zeigen und damit beginnen, in Kategorien nachhaltiger Entwicklung zu denken und zu planen, ist die sozialökologische Seite wirtschaftlichen Handelns nicht nur unterentwickelt, sondern es zeichnet sich gar ein Rückschritt gegenüber den in langfristigen historischen Lernprozessen schrittweise definierten und durchgesetzten wirtschaftsethischen Standards ab.

\section{Der soziologische Auftrag an die Ärzte}

Ärzte sind, viel mehr als die oft wenig geliebten Soziologen, eine weithin geschätzte und mit gesellschaftlicher und moralischer Autorität ausgestattete Berufsgruppe.

Sie könnten und sollten diese Autorität nutzen, um die von ihnen diagnostizierten und behandelten Leiden dort, wo sie deutlich auf gesellschaftliche Faktoren und wirtschaftliche Ursachen hinzuweisen scheinen, auch als solche zu benennen und öffentlich zu debattieren. Sie könnten zu einem verstärkten politischen Problembewusstsein in diesen Fragen beitragen und gewissermassen, als Anwälte ihrer Patienten, jene gesellschaftlichen und wirtschaftlichen Bedingungen anklagen, an denen die Menschen an «Leib und Seele» leiden. Schon dies wäre ein wertvoller Beitrag zu einem verantwortlichen Umgang mit den Opfern der skizzierten gesellschaftlichen Umbrüche, die in ihrer Isolation oft nur Selbstzweifel und Selbstvorwürfe oder den stigmatisierenden Blick ihrer Umgebung ernten: «Nicht verlachen, nicht bemitleiden, nicht verabscheuen, sondern verstehen...» - diese spinozistische Moral könnte nicht zuletzt dank eines Engagements der Ärzte in den öffentlichen Debatten der Gegenwart wieder neu belebt werden.

\section{Literatur \\ 1 Boltanski L, Chiapello E. Der neue Geist des Kapitalismus. Konstanz: Universitätsverlag; 2003. \\ 2 Weber M. Gesammelte Aufsätze zur Wissenschaftslehre. Tübingen; 1973}

\section{Prof. Dr. Franz Schultheis}

Département de sociologie

Université de Genève

Bd du Pont d'Arve 40

$\mathrm{CH}-1211$ Genève 4

Franz.Schultheis@socio.unige.ch 